\title{
Material Optimisation for Optical Data Storage
}

\author{
Samuel Guerin ${ }^{1}$, Majid Hassan ${ }^{1}$, Brian Hayden ${ }^{1,3}$, D W Hewak ${ }^{1}$, Graeme Purdy ${ }^{2}$ and Robert E Simpson ${ }^{1}$ \\ ${ }^{1}$ Optoelectronics Research Centre and School of Chemistry, University of Southampton, Southampton, SO17 IBJ \\ ${ }^{2}$ Ilika Technologies Ltd, Centre for Enterprise and Innovation, University of Southampton, Southampton, SO17 IBJ \\ ${ }^{3}$ Corresponding author: beh@.soton.ac.uk
}

\begin{abstract}
High throughput synthesis and screening methodologies of optical data storage materials have allowed an in depth study of more than 4000 compositions. We report on the optical and electrical compositional dependence of these materials.

OCIS codes: (210.0210) Optical data storage; (210.4590) Optical disks (310.6860) Thin films, optical properties;
\end{abstract}

\section{Introduction}

This presentation introduces a method of high throughput combinatorial deposition and screening of chalcogenide based alloys for optical data storage. Optical phase change data storage materials are required to be: fast switching, re-writeable, have high optical contrast between phases, have long archival stability and allow ever decreasing amorphous mark dimensions written within. This large parameters space lends itself to optimisation via high throughput methodology. In this work, the results of a number of sensitive and fast characterisation measurements developed for thin film materials are reported together with high throughput measurement of the films' optical and electrical characteristics.

Our initial attempts to increase the efficiency of the material deposition process were to fabricate varied composition chalcogenide films. This was achieved through exploitation of the natural composition variance of films deposited via the pulsed laser deposition (PLD) method[1]. However the technique lacked control and was limited to compositions close to that of the target material. Hence a new technique was required capable of controlled deposition over wide composition. The chosen MBE technique allows simultaneous graded elemental deposition over an entire substrate with complete control of the resultant film's compositional[2].

\section{Results and Figures}

To date we have synthesised 40 samples over a variety of compositional ranges. Each sample has at least 100 discrete measurement points. Figure 1 shows how the combination of just five synthesised samples largely covers the complete range of ternary compositions of Ge:Sb:Te.

High throughput analysis techniques have been developed to characterise the optical, electrical and structural properties of the wide ranging compositions deposited. Figure 2 shows an optically processed image of an asdeposited Ge:Sb:Te film. As-deposited crystalline compositions are clearly visible by the lighter region in the bottom left hand corner of the image (Also confirmed by XRD). This analysis tool can be used to identify compositions which might show potential for initialisation free manufacturing processes.

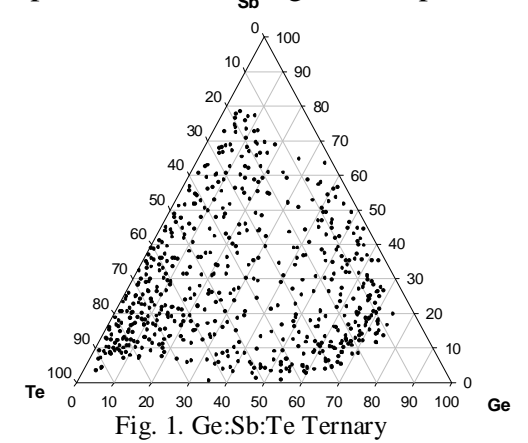

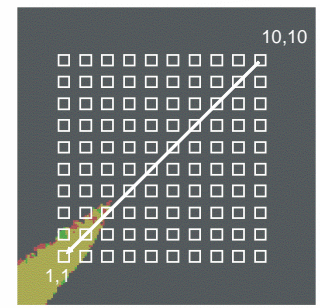

Fig. 2. Digitally processed image of as deposited film

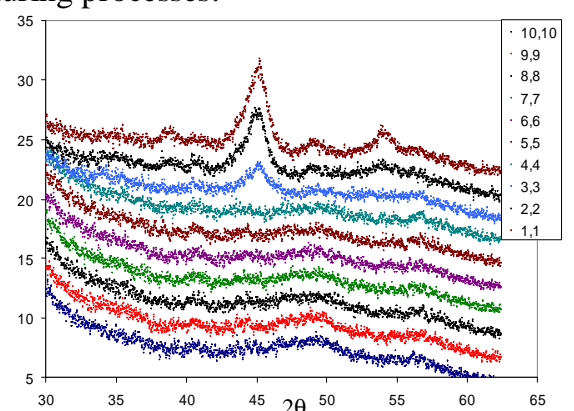

Fig. 3. XRD spectral showing crystalline region

Novel chalcogenide systems are now being explored for both electrical and optical phase change data storage. The high throughput deposition methodology could also be applied to explore optimum compositions for holographic data storage materials. The variety of potential chalcogenide phases available and even wider range of doped materials, the flexibility and controllability of the high throughput synthesis makes this combinatorial technique an ideal for the discovery of new storage materials.

\section{References}

[1] Gill, D.S. et. al. 'Characterisation of Ga-La-S chalcogenide glass thin film optical waveguides, fabricated by pulsed laser deposition’ J. Non Cryst. Solids 191(3) 321- 326 (1995)

[2] Guerin, S. et. al. 'Combinatorial Electrochemical Screening of Fuel Cell Electrocatalysts' J. Comb. Chem. 6(1)

149-158. (2004) 